\title{
MEASURES OF GALACTIC AND INTERGALACTIC MASS IN CLUSTERS
}

\author{
D. WINDRIDGE, S. PHILLIPPS, M. BIRKINSHAW \\ Astrophysics Group, University of Bristol
}

\section{Estimation of the Electron Gas Mass}

If a galaxy cluster's X-ray gas distribution follows an isothermal polytropic $\beta$ model, we may write the electron radial density distribution as; $n_{e}=n_{e 0}\left(1+r^{2} / r_{c}^{2}\right)^{-3 / 2 \beta}, r_{c}$ being the core radius and $n_{e 0}$ the central electron density. This may be related to both an X-ray surface brightness distribution and a Sunyaev-Zel'dovich effect distribution (Sarazin 1986). Fitting to observational data then enables us to constrain the value of $\beta$. The normalisation value, $n_{e 0}$, to obtain a total mass estimate is calculated via the relationship between the $\mathrm{X}$-ray and $\mathrm{S}-\mathrm{Z}$ distribution normalisation constants, and the gas temperature and spectral emissivity parameters from fits to the X-ray spectrum. We are then in a position to evaluate $n_{e}(r)$ and its integral; the total electron gas mass. If we can further assume that there exists a simple ratio between the electron and proton number densities within the gas, we may straightforwardly posit a value for the total gas mass. An additional method of determining the polytropic gas index exists, with optical constraints on the galactic velocity dispersion, through the relation; $\beta=\mu m_{H} \sigma_{z}^{2} / k_{B} T_{e}$. Studies at optical, as well as X-ray and radio wavelengths are thus useful as a corroborative measure in determining the total gas mass.

\section{Estimation of the Baryonic Galactic Mass}

In formulating the total baryonic galactic mass, we shall follow Persic \& Salucci (1992) in treating the galactic mass-to-light ratio as a powerlaw with respect to luminosity; i.e., $\Gamma(L)=A\left(L / L_{\star}\right)^{\eta}$. Combining this with the Schechter (1976) luminosity function, we may derive a classspecific estimate of the total field density across the entire luminous range: 
$\rho_{b}=\phi_{\star} L_{\star} A \int_{x_{\min }}^{x_{\max }} x^{1+\alpha+\eta} e^{-x} d x$, with $\mathrm{x} \equiv \frac{L}{L_{\star}}$, and $\phi_{\star} \& L_{\star} ;$ Schechter normalisation parameters. The value $\eta$ is not subject to rigorous observational constraint, but we shall argue for a value of $\sim-0.1$ by noting that the galactic stellar $\mathrm{M} / \mathrm{L}$ ratio is $\approx 1 \frac{M_{\odot}}{L_{\odot}}$, practically across the entire observational range (Longmore 1982); whilst the HI mass remains roughly constant. The baryonic $\mathrm{M} / \mathrm{L}$ ratio can then only rise modestly with decreasing luminosity, the excess baryons being supplied by the increasing HI gas mass fraction. Since the density equation is critically sensitive to the sum $\alpha+\eta$ for values around -1.8 (i.e., $\alpha \sim-1.7$ ), below which the integral diverges with decreasing $x_{\min }$, and since this is so close to dwarf galaxy LF slope indicated by deep cluster surveys, we shall treat the dwarf and giant galaxies as discrete Schechter populations (Driver et al. 1994). Evaluating the mass integral separately for the two classes, then, we find a field dwarf/giant mass density ratio of 2.5:1. In a flux-limited cluster survey, in which only the giants are reliably detected, a total baryonic mass estimate may then be obtained through the introduction of a simple multiplying factor.

\section{Estimation of the Total Galactic Mass in Clusters}

Any estimate of the total galactic mass in clusters proceeding in the manner set out above must incorporate some consideration of the variation of dark matter mass with luminosity, paying particular regard to the mass-to-light ratios at the dwarf luminosities, where most of the matter would appear to be concentrated. Perhaps the best indication of dwarf mass-to-light ratios at very faint luminosities comes from the recent survey of the local group dSphs by Irwin \& Hatzidimitriou (1995), suggesting a $L^{-.965}$ powerlaw operating over the survey range. Since it would be unphysical for this power-law to extend much beyond the survey luminosity limits, we envisage a composite power-law for the general dwarf $\Gamma$ function, with a baryonic $\left(L^{-0.1}\right)$ power-law taking-over from the $\eta=-.965 \mathrm{dSph}$ behaviour at luminosities greater than $10^{6.7} L_{\odot}$. Performing the density integration within each of the two domains of behavior then gives a dwarf/giant density ratio of 5.5:1 allowing an estimate of the total galactic mass in clusters from the giant galaxy number counts alone.

\section{References}

Birkinshaw, M. \& Hughes, J. P., 1994, Astrophys. J. 420, 33

Driver S. P. et al., 1994, Mon. Not. R. astron. Soc. 266, 155

Irwin M. \& Hatzidimitriou D., 1995, Mon. Not. R. astron. Soc. 277, 1354

Longmore A. J. et al., 1982, Mon. Not. R. astron. Soc. 200, 325

Persic M. \& Salucci P., 1992, Mon. Not. R. astron. Soc. 258, 14P

Sarazin C. L., 1986, Rev. Mod. Phys. 58, 1

Schechter P. L., 1976, Astrophys. J. 203, 297 